\title{
Ten-User Truly Asynchronous Gigabit OCDMA Transmission Experiment With a 511-Chip SSFBG En/Decoder
}

\author{
Taro Hamanaka, Xu Wang, Member, IEEE, Naoya Wada, Member, IEEE, \\ Akihiko Nishiki, Member, IEEE, and Ken-ichi Kitayama, Fellow, IEEE
}

\begin{abstract}
A ten-user truly asynchronous gigabit coherentoptical-code-division-multiple-access (OCDMA) transmission was experimentally demonstrated without using any timing coordination. The enabling technologies are a record-length 511-chip superstructured-fiber-Bragg-grating (SSFBG) en/decoder and a supercontinuum (SC)-based optical-thresholding technique to significantly suppress the signal interference beat noise as well as the multiple-access-interference (MAI) noise.
\end{abstract}

Index Terms-Asynchronous transmission, beat noise, fiber Bragg grating (FBG), multiple access interference (MAI), optical code division multiple access (OCDMA), optical thresholding, supercontinuum.

\section{INTRODUCTION}

$\mathbf{O}$ PTICAL access networks have been developed at a rapid pace, particularly in Japan, where fiber-to-the-home (FTTH) service provisioning has gained momentum; and it is expected that 30 million customers will switch from plain old telephone service (POTS) to FTTH by 2010 [1]. Although several network topologies can be used for FTTH, including single star (SS), active double star (ADS), and passive double star (PDS), PDS is generally used because of its low-cost and flexible configuration. In a PDS network, a passive splitter is placed between the optical line terminal (OLT) in the central office and the optical network unit (ONU) at each user's location. Each ONU (i.e., user) shares an optical fiber between the OLT and a splitter. A PDS network is also called a passive optical network (PON) [1], [2].

There are various types of PONs, with each type depending on the signal-multiplexing technique used. In time division multiple access (TDMA) utilizing a time-division-multiplexing (TDM) technique, $N$ time slots are assigned to $N$ users. However, the increase of bit rate is limited by the bandwidth limitations of the electronics, and only synchronous networks

Manuscript received July 1, 2005; revised October 24, 2005.

T. Hamanaka and K. Kitayama are with the Department of Information and Communication Technology, Osaka University, Osaka 565-0871, Japan (e-mail: hamanaka@ac.eie.eng.osaka-u.ac.jp; kitrayama@comm.eng.osaka-u. ac.jp).

X. Wang and N. Wada are with the Ultrafast Photonic Network Group, National Institute of Information and Communication Technology, Tokyo, Japan (e-mail: xwang@nict.go.jp; wada@nict.go.jp).

A. Nishiki was with Oki Electronics Industry Company, Ltd., Tokyo, Japan. $\mathrm{He}$ is now with the New Energy and Industrial Technology Development Organization, Kanagawa, Japan.

Digital Object Identifier 10.1109/JLT.2005.861126 can be configured. In wavelength division multiple access (WDMA), a wavelength is assigned to each user; so bandwidth is used efficiently, and asynchronous transmission is supported [1]-[3]. However, the presently available coarse-wavelengthdivision-multiplexing (CWDM) technique supports only 18 wavelengths; so it is not well suited to existing PON systems, which normally has up to 32 users. In addition, increasing the number of users increases the equipment costs. A promising approach to reducing the cost in next-generation access systems is optical code division multiple access (OCDMA) due to its simple ONU and OLT configurations, which require no electronics for synchronization. Moreover, attractive features of OCDMA include all-optical processing (which means the en/decoding operation is performed in the optical domain), truly asynchronous transmission, bandwidth efficiency, soft capacity on demand, protocol transparency, simplified network control, and flexibility of controlling the quality of service (QoS) [1], [4]-[8].

The several different OCDMA schemes that have been proposed can be roughly classified according to their operation principles as either incoherent or coherent OCDMA [7]-[9]. These OCDMA schemes, which use various coding and detection schemes, have been investigated since the mid-1980s [10]. Recently, coherent OCDMA using ultrashort optical pulse has been receiving increasing attention due to the advances in reliable and compact en/decoder devices and detection schemes [7]-[9], [11]-[15].

In this paper, we present a ten-user truly asynchronous gigabit coherent-OCDMA transmission experiment demonstrated for the first time. The enabling techniques are a record-length 511-chip superstructured-fiber-Bragg-grating (SSFBG) en/decoder and a supercontinuum (SC)-based opticalthresholding technique to significantly suppress the signal interference beat noise as well as multiple-access-interference (MAI) noise.

\section{OCDMA-PON SYSTEM}

In OCDMA systems, a different optical code (OC) is assigned to each user, and the central office has all of the OC information. The architecture of a common OCDMA-PON system is shown in Fig. 1. Direct connections between users within the network are not assumed in this architecture. In the downlink, the central office encodes the signal using the code assigned to the destination user, so only that user can decode 


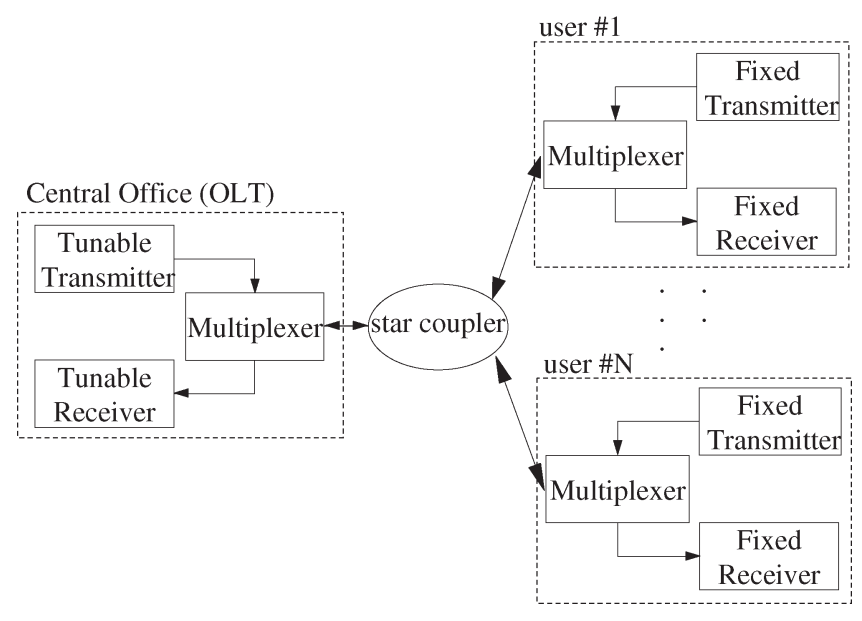

Fig. 1. OCDMA network architecture.

the signal. In the uplink, all users can simultaneously transmit signals to the central office at anytime because each has a different OC [5].

As mentioned above, OCDMA schemes are classified based on their operation principle as either incoherent or coherent OCDMA. Incoherent schemes, which work on an intensity basis, process coding in a unipolar $(0,1)$ manner. Coherent ones, which work on a field amplitude basis, process coding in a bipolar $(-1,+1)$ manner. In terms of correlation property, coherent schemes are better and have been used in recent OCDMA research and development [7], [9], [11]-[17]. The en/decoders that can generate bipolar code include planar lightwave circuit (PLC) [7], [16] and SSFBG. SSFBG en/decoders can simplify the network system entirely and reduce network costs [4], [9], [12], [14], [17], [18].

The performance of OCDMA systems is degraded by MAI and beat noises [19]-[21]. Here, the MAI and beat noises refer to the incoherent and coherent part of the noises, respectively, as discussed in [21]. Therefore, a way to overcome these drawbacks is as essential as the key technologies. The use of en/decoders with ultralong code [20], time gating [7], [22], and optical thresholding [11]-[14], [19], [20], [23] have been proposed to reduce the MAI noise in OCDMA systems. Longer code and synchronized OCDMA can be used to reduce beat noise. However, in synchronized OCDMA, the signal and each interference have to be precisely aligned to avoid the overlap between them. Therefore, in practice, it is almost impossible to control the optical path within chip-length order through the network [21]. Moreover, it requires timing coordination in the system and sacrifices the asynchronism of the OCDMA. For a practical OCDMA system, "asynchronism" is essential. Using longer code is an effective means to reduce the beat noise as well as the MAI noise. In this report, we use a 511-chip SSFBG, which exhibits the longest code presently [18].

\section{Key TeChNOLOGIES}

\section{A. 511-Chip SSFBG En/Decoder}

An SSFBG is an FBG in which the refractive index modulation varies rapidly and the refractive index modulation profile

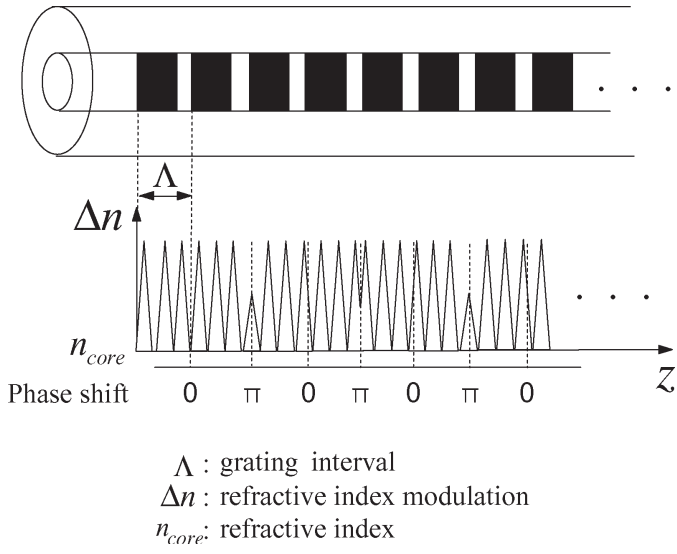

Fig. 2. Structure of the phase-shifted SSFBG.

varies slowly along its length; that is, it functions as a series of various uniform FBGs. SSFBGs can be designed and fabricated with a refractive index modulation profile inserting phase shifts ( 0 or $\pi$ ) between different segments, as shown in Fig. 2. Such a phase-shifted SSFBG can generate a series of coherent chip pulses by injecting a short optical pulse. The phase of the pulses is determined by the pattern of the phase shifts. Therefore, SSFBG en/decoders can generate a bipolar code and provide a good auto/cross correlation property [9], [17], [18]. The correlation property gets better with the longer code length. Using a holographic technique to fabricate SSFBGs enables them to be fabricated with a long code and a variable code pattern [18].

For our experiment, we fabricated the 511-chip binary-phaseshift-key (BPSK) SSFBGs with a Gold-code pattern and a chip-rate as high as $640 \mathrm{Gchip} / \mathrm{s}$. The reflection spectrum for a 511-chip SSFBG is shown in Fig. 3(a). Fig. 3(b) and (c) show the second-harmonic-generation (SHG) trace and the waveform of the input pulse, and Fig. 3(d) shows the waveform of the encoded signal. The pulses with a width equal to the chip duration $(\sim 1.8 \mathrm{ps})$ were spread over the bit interval of 800 ps. Fig. 3(e) and (f) show the auto- and cross-correlation waveforms after the SSFBG decoder. These results show better correlation properties than the other en/decoders, such as PLC [9], [18]. They are independent of the polarization state of the input signal, indicating that the SSFBGs were fabricated well [18]. These are the longest code so far, enabling us to challenge a truly asynchronous OCDMA.

\section{B. SC-Based Optical Thresholding}

The existence of MAI noise and beat noise in multiuser OCDMA networks severely limits the maximum number of active users [20], [21]. As mentioned above, time gating can be used to reduce MAI noise. The idea is to open the time window the moment the autocorrelation main lobe passes [7], [22]. However, an OCDMA system with timing coordination does not feature asynchronous transmission. A promising approach to overcoming this problem is to apply optical thresholding, which only uses passive optical devices for asynchronous OCDMA systems. Optical thresholding has been used in multiuser OCDMA experiments, such as SHG in periodically 
(a)

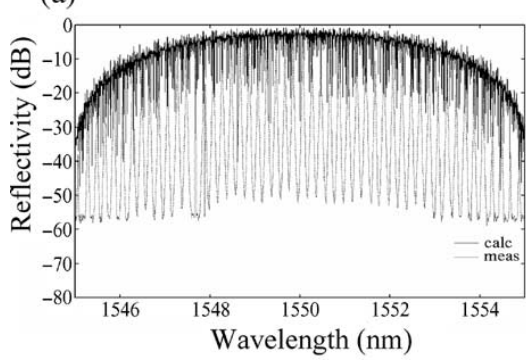

(c)
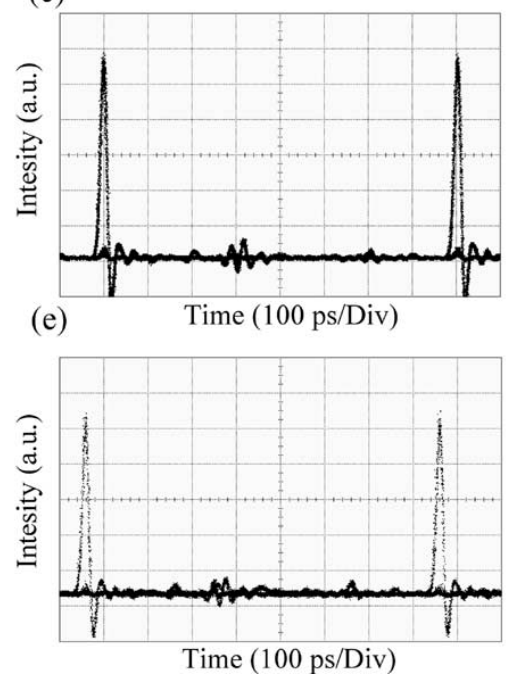

(b)

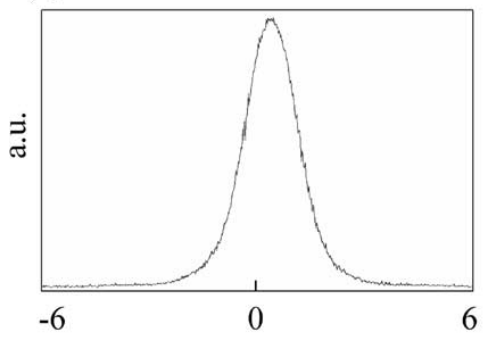

(d)

Time (ps)
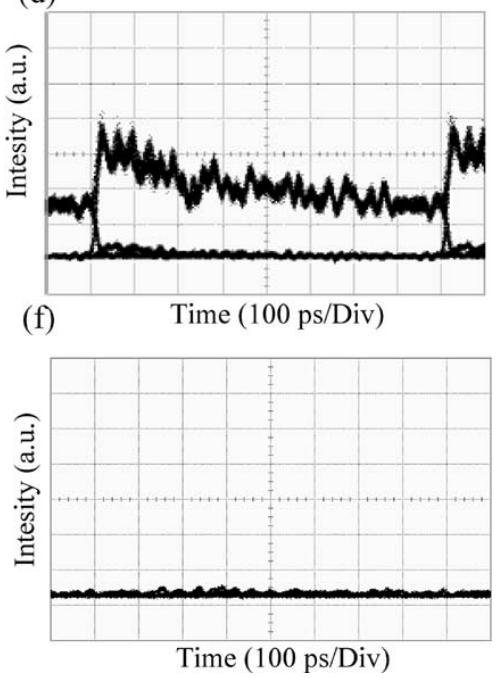

Fig. 3. Experimental results on a 511-chip SSFBG. (a) Reflection spectrum, (b) SHG trace of the input pulse, and the waveforms of (c) input pulse, (d) encoded signal, (e) autocorrelation, and (f) cross correlation.

poled lithium niobate (PPLN) [11], nonlinear optical loop mirror (NOLM) [12], nonlinear effect in dispersion shift fiber [19], [20], high nonlinear fiber (HNLF) [13], [23], and holey fiber [14].

We propose optical thresholding based on SC generation in dispersion flattened fiber (DFF) to suppress MAI noise effectively. SC is a phenomenon in which the bandwidth of an optical short pulse broadens over more than $100 \mathrm{~nm}$ [24]. The proposed SC-based optical thresholder is shown in Fig. 4. It is composed of a 2-km DFF and a 5-nm optical bandpass filter (OBPF). Specifications of DFF include zero-dispersion wavelengths of 1523.1/ 1575.2 nm [25] and an Aeff of about 24. The operating principle is that the EDFA boosts the decoded signal to a proper level, which has a well-defined shape, with $\mathrm{a} \sim 2$-ps pulsewidth [shown in Fig. 3(b)]. A signal with high peak power can generate SC in the DFF, while incorrectly decoded signals (MAI noise) with low peak power are spread over a long time span and are thus unable to generate SC. The OBPF only passes SC signals and rejects the original signal. Therefore, after a bandpass filter (BPF), the correctly decoded signal can be recovered without the MAI noise. However, beat noise cannot be suppressed by optical thresholding and is thus a critical issue limiting system performance. The only effective method for reducing beat noise is to use a longer code [21]. Thus, a ten-user-truly asynchronous OCDMA system can be realized by using 511-chip SSFBG en/decoders and optical thresholding.

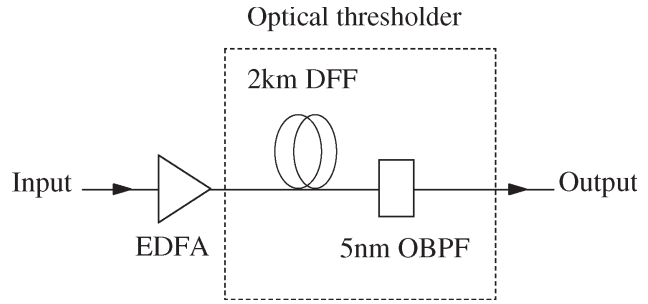

Fig. 4. SC-based optical thresholder.

\section{TEN-USER TRULY-ASYNCHRONOUS OCDMA TRANSMISSION EXPERIMENT}

Fig. 5 shows our experimental setup for demonstrating a ten-user truly asynchronous OCDMA transmission. An optical pulse train with a pulsewidth of about 1.8 ps was generated by a mode-locked laser diode (MLLD). The center wavelength was $1550 \mathrm{~nm}$, and the repetition rate was $10 \mathrm{GHz}$. The train was converted to a $2^{3}-1$ pseudorandom bit sequence (PRBS) at $1.25 \mathrm{~Gb} / \mathrm{s}$ by $\mathrm{LiNbO}_{3}$ intensity modulators (LN-IM). Its eye diagram is shown in Fig. 5(a). The amplified optical signal was equally split into ten arms and encoded using ten different SSFBG encoders. Fig. 5(b) shows the signal encoded by encoder 1 . These encoders (code 1-10) were designed to have a relatively low cross-correlation level. A tunable attenuator (ATT) with a switch was inserted into each arm to balance the optical power levels across the arms and to adjust the number of 
(a)

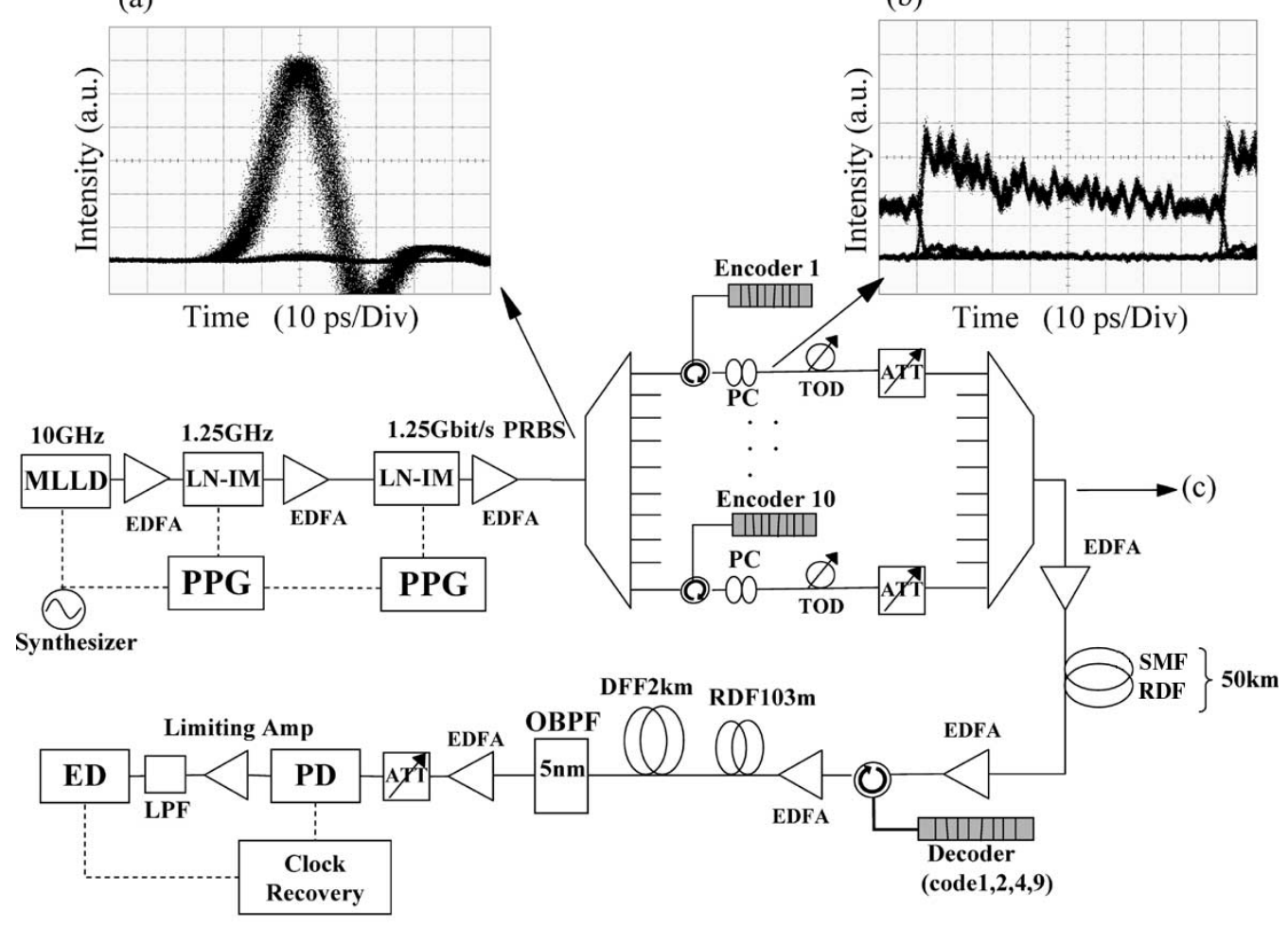

(c)

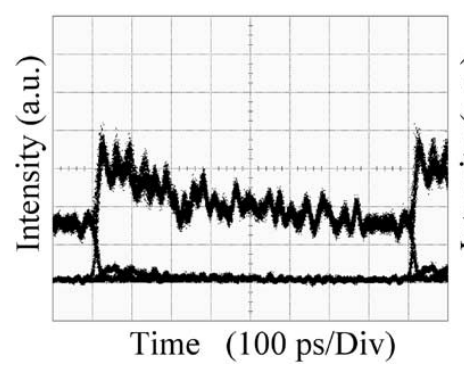

5 users

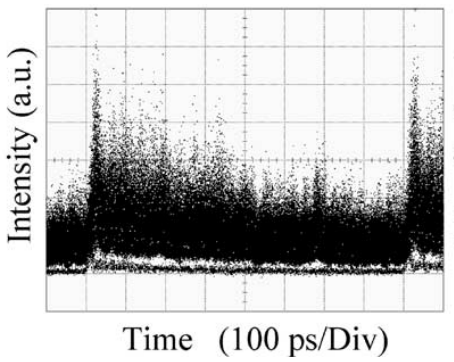

10 users

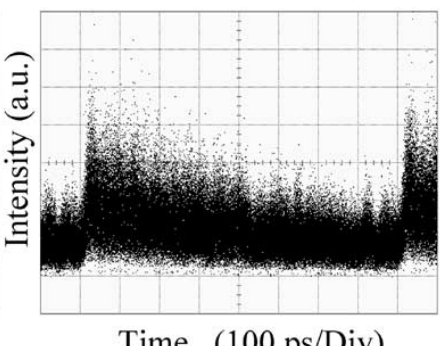

Fig. 5. Setup of the OCDMA transmission experiment.

active users. Tunable optical delay lines (TODL) were used to investigate the impact of different phases of signal-interference overlapping. Polarization controllers (PC) were used to investigate the system performance in the worst case scenario in which the polarization states of all signals are aligned. These encoded signals were mixed [as shown in Fig. 5(c)] for different numbers of users, amplified, and launched into a single mode fiber (SMF) and a reverse dispersion fiber (RDF). On the receiver side, the amplified signal was decoded by an SSFBG decoder.

We prepared four decoders (code 1, 2, 4, 9). Fig. 6(a) shows the eye diagrams of the decoded signals for different numbers of users for decoder 1 . As the number increased, the MAI and beat noises became more prominent. Therefore, we employed optical thresholding by using SC generation in the DFF for the decoded signal with low signal-to-noise ratio (SNR). The amplified signal was launched into a 103-m RDF to compensate for the chirp and then into the DFF. The pulse, which had a well-defined shape with $\mathrm{a} \sim 2$-ps pulsewidth and high peak power, was able to generate an SC in the DFF. In contrast, the MAI noise with low peak power was unable to generate SC, which remained in the vicinity of $1550 \mathrm{~nm}$. Table I shows the power input into the DFF for different numbers of users for decoder 1 . After the DFF, the OBPF cuts out only the signal component. Fig. 6(b) shows the eye diagrams of the signal after the OBPF. Fig. 7 shows the spectra of the decoded signal after the DFF and after the OBPF for different numbers of users. As shown in Fig. 7, the band cutout by the OBPF approached $1550 \mathrm{~nm}$ as the number of users increased. This resulted from SNR degradation. Finally, the signal was data-rate detected by an optical packet receiver, followed by a 7.8-GHz low-pass filter (LPF), and measured by an error detector (ED).

The bit error rates (BERs) shown in Fig. 8 were measured with different numbers of users for both (a) back-to-back (B-to-B) and (b) 50-km transmissions. In the B-to-B experiment, the 40-m SMF was replaced with an RDF before the DFF. In the worst case scenario for OCDMA networks, errorfree transmission in the ten-user case was achieved for the four decoders. Fig. 9 shows the average power penalty against the number of users for a BER of $10^{-9}$ together with the theoretical calculations. Formulas that have been developed in [21] have 

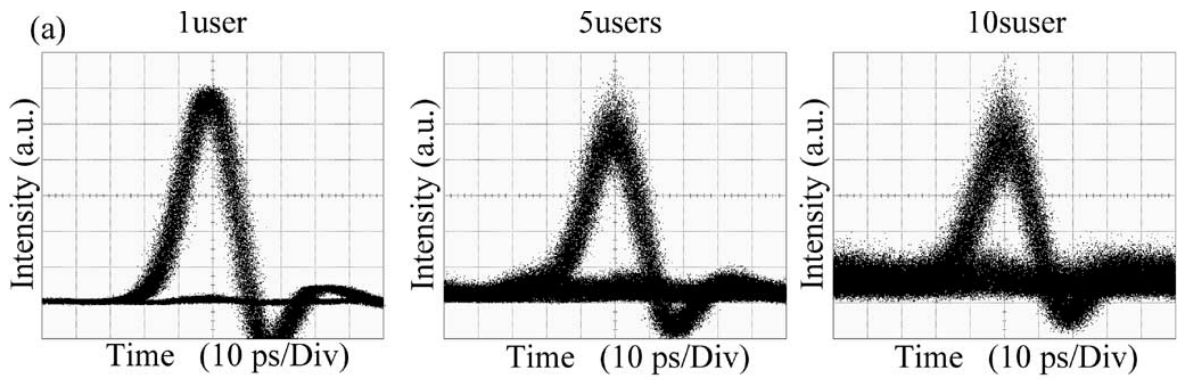

(b)
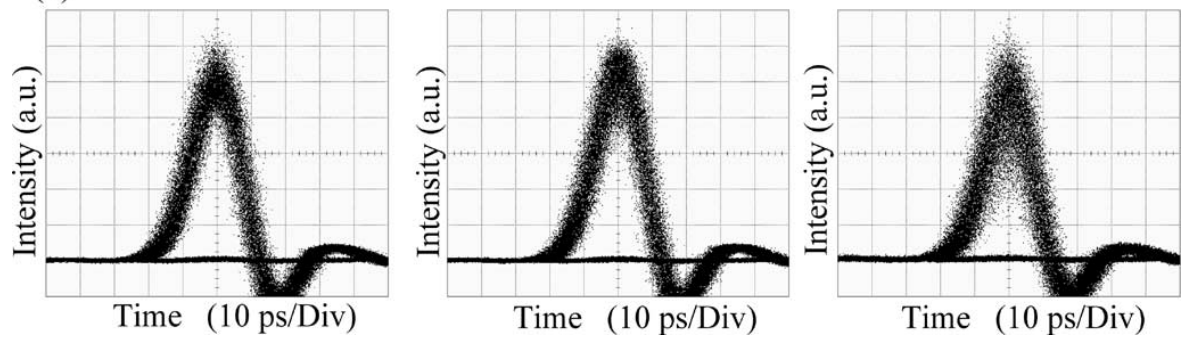

Fig. 6. Measured eye diagrams (a) after decoder 1 and (b) after the OBPF.

TABLE I

OPTICAL POWERS INTO THE DFF

\begin{tabular}{|c|c|c|c|c|c|c|}
\hline $\begin{array}{c}\text { Number of } \\
\text { users }\end{array}$ & 1 & 3 & 5 & 8 & 9 & 10 \\
\hline $\begin{array}{c}\text { Input power } \\
(\mathrm{dBm})\end{array}$ & 1.68 & 4.13 & 6.49 & 7.67 & 8.65 & 10.75 \\
\hline
\end{tabular}
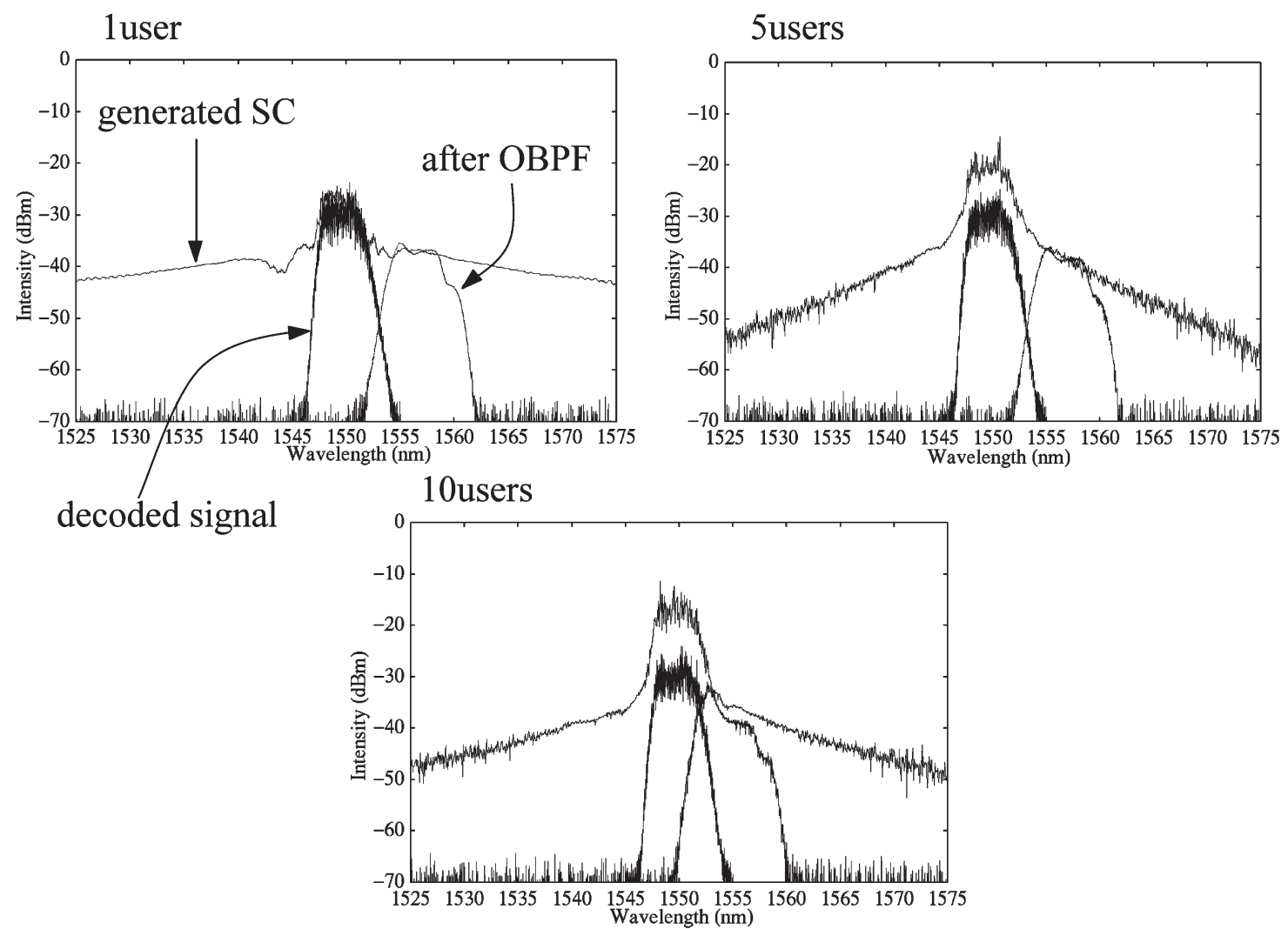

Fig. 7. Measured spectrums of the decoded signal, generated SC, and the signal after OBPF.

been used here for theoretical calculation. In the calculation, an average interference level of $\xi \approx-27 \mathrm{~dB}$ and an ideal optical thresholder that can eliminate the MAI noise outside the autocorrelation peak completely are assumed. In the case without the optical thresholder, the experimental results agree with the theoretical calculations very well. In the case with 
(a)

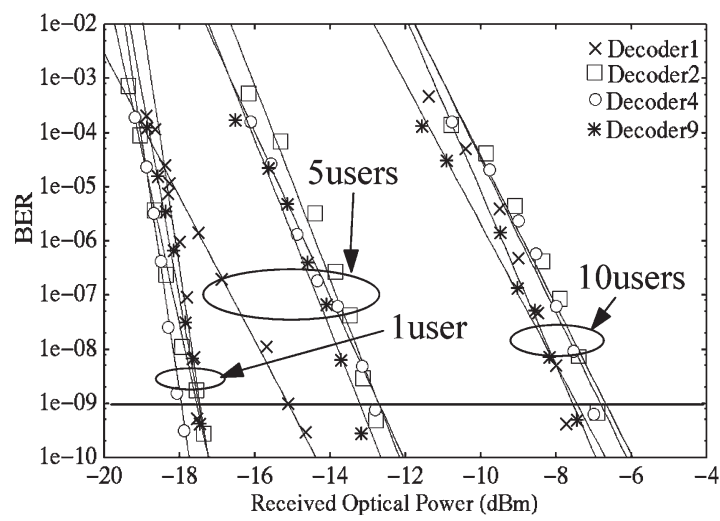

Fig. 8. Measured BERs of (a) back-to-back and (b) 50-km transmissions.

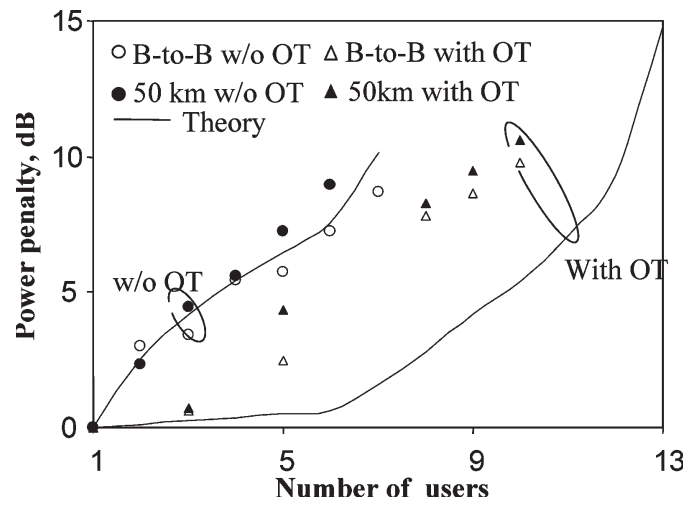

Fig. 9. Power penalty versus the number of users.

the optical thresholder, the deviations between the experimental and theoretical results were mainly due to the nonideal optical thresholding, which means further improvement of the optical thresholding will enable us to accommodate more users.

Although we first measured the BER in the worst signalmultiplexing case possible, we also measured it in two other cases: (case 1) the same polarization states and random time delays and (case 2) random polarization states and time delays. The average powers were the same. Fig. 10 shows the results and the original case (the worst case scenario) for the ten-user case and decoder 1 . In all these cases, an error-free transmission was achieved. These results show that the power penalty of the original case was the highest.

In addition, we also measured the BER without the SCbased optical thresholding for B-to-B and 50-km transmissions with random polarization states and time delays. As shown in Fig. 10, an error-free transmission was not achieved for ten users but for at most seven users (B to B) and at most six users $(50 \mathrm{~km})$. As shown in Fig. 9, the experimental results agree well with the theoretical results, indicating that the 511-chip SSFBG en/decoders have good correlation properties. Moreover, the success of this ten-user experiment is due to the use of SCbased optical thresholding.

\section{CONCLUSION}

A ten-user truly asynchronous OCDMA transmission experiment has been demonstrated at $1.25 \mathrm{~Gb} / \mathrm{s}$ for the first (b)
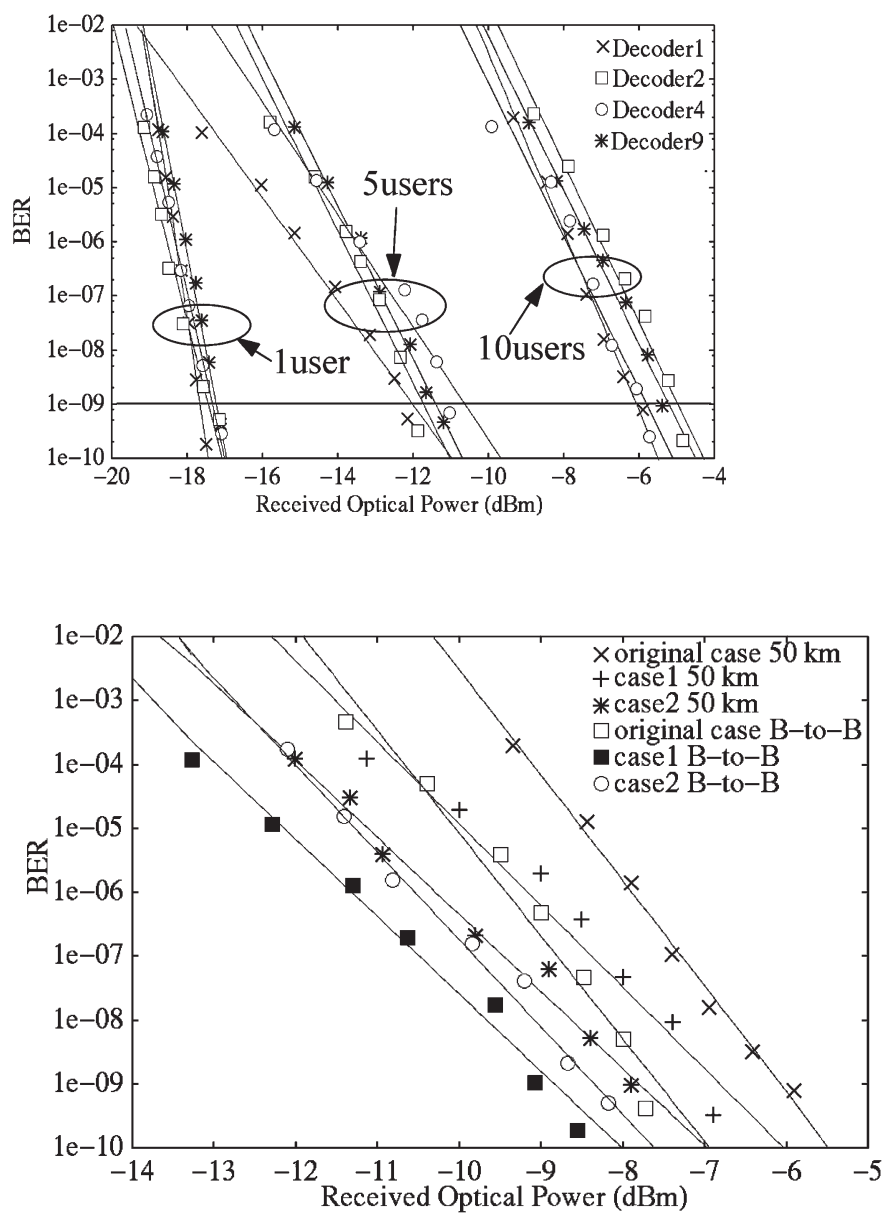

Fig. 10. Measured BERs in each signal-multiplexing case.

time, where we investigated several possible cases in practical OCDMA systems by using PCs and TODLs. An errorfree transmission was achieved for four decoders for all cases without any timing coordination due to the use of the 511-chip SSFBG en/decoders as well as SC-based optical thresholding. Further improvements in these devices will further enhance the multiuser high-speed access capabilities of OCDMA systems.

\section{ACKNOWLEDGMENT}

The authors would like to thank Y. Tomiyama, T. Hanyu, T. Makino, and H. Sumimoto of the National Institute of Information and Communications Technology (NICT) for their support.

\section{REFERENCES}

[1] K. Kitayama, X. Wang, and H. Sotobayashi, "Gigabit-symmetric FTTH-OCDMA over WDM PON," in Proc. 9th Conf. Optical Network Design \& Modelling (ONDM), Milan, Italy, Feb. 2005, pp. 273-281.

[2] M. Nakamura, H. Ueda, S. Makino, T. Yokotani, and K. Oshima, "Proposal of networking by PON technologies for full and Ethernet services in FTTx," J. Lightw. Technol., vol. 22, no. 11, pp. 2631-2640, Nov. 2004.

[3] S.-J. Park, C.-H. Lee, K.-T. Jeong, H.-J. Park, J.-G. Ahn, and K.-H. Song, "Fiber-to-the-home services based on wavelength-division-multiplexing passive optical network," J. Lightw. Technol., vol. 22, no. 11, pp. 25822591, Nov. 2004. 
[4] K. Kitayama, X. Wang, and H. Sotobayashi, "State of the art of OCDMA, OCDM, and OC-label switchings," in Proc. 30th Eur. Conf. Optical Communication (ECOC), Stockholm, Sweden, Sep. 2004, pp. 266-269.

[5] A. Stock and E. H. Sargent, "The role of optical CDMA in access networks," IEEE Commun. Mag., vol. 40, no. 9, pp. 83-87, Sep. 2002.

[6] J. A. Salehi, "Code division multiple-access techniques in optical fiber networks-Part I: Fundamental principles," IEEE Trans. Commun., vol. 37, no. 8, pp. 824-833, Aug. 1989.

[7] K. Kitayama, H. Sotobayashi, and N. Wada, "Optical code division multiplexing (OCDM) and its applications to photonic networks," IEICE Trans. Fundam., vol. E82-A, no. 12, pp. 2616-2626, Dec. 1999.

[8] M. E. Marhic, "Coherent optical CDMA networks," J. Lightw. Technol., vol. 11, no. 5/6, pp. 854-863, May/Jun. 1993.

[9] X. Wang, K. Matsushima, A. Nishiki, N. Wada, and K. Kitayama, "High reflectivity superstructured FBG for coherent optical code generation and recognition," Opt. Express, vol. 12, no. 22, pp. 5457-5468, Nov. 2004.

[10] P. R. Prucnal, M. A. Santoro, and T. R. Fan, "Spread spectrum fiberoptic local area network using optical processing," J. Lightw. Technol., vol. LT-4, no. 5, pp. 547-554, May 1986.

[11] Z. Jiang, D. S. Seo, S. D. Yang, D. E. Leaird, R. V. Roussev, C. Langrock, M. M. Fejer, and A. M. Weiner, "Four user $10 \mathrm{~Gb} / \mathrm{s}$ spectrally phase-coded O-CDMA system operating at $\sim 30 \mathrm{fj} /$ bit," IEEE Photon. Technol. Lett., vol. 17, no. 3, pp. 705-707, Mar. 2005 .

[12] J. H. Lee, P. C. Teh, P. Petropoulos, M. Ibsen, and D. J. Richardson, "A grating-based OCDMA coding-decoding system incorporating a nonlinear optical loop mirror for improved code recognition and noise reduction," J. Lightw. Technol., vol. 20, no. 1, pp. 36-46, Jan. 2002.

[13] K. Li, W. Cong, V. J. Hernandez, R. P. Scott, J. Cao, Y. Du, J. P. Heritage, B. H. Kolner, and S. J. B. Yoo, "10 Gb/s optical CDMA encoderdecoder BER performance using HNLF thresholder," presented at the Optical Fiber Communication Conf. (OFC), Los Angeles, CA, 2004, Paper MF87.

[14] J. H. Lee, P. C. Teh, Z. Yusoff, M. Ibsen, W. Belardi, T. M. Monro, and D. J. Richardson, "A holey fiber-based nonlinear thresholding device for optical CDMA receiver performance enhancement," IEEE Photon. Technol. Lett., vol. 14, no. 6, pp. 876-878, Jun. 2002.

[15] R. P. Scott, W. Cong, K. Li, V. J. Hernandez, B. H. Kolner, J. P. Heritage, and S. J. B. Yoo, "Demonstration of an error-free $4 \times 10 \mathrm{~Gb} / \mathrm{s}$ multiuser SPECTS O-CDMA network testbed," IEEE Photon. Technol. Lett., vol. 16, no. 9, pp. 2186-2188, Sep. 2004

[16] K. Kitayama and N. Wada, "Photonic IP routing," IEEE Photon. Technol. Lett., vol. 11, no. 12, pp. 1689-1691, Dec. 1999.

[17] P. C. Teh, P. Petropoulos, M. Ibsen, and D. J. Richardson, "A comparative study of the performance of seven- and 63-chip optical codedivision multiple-access encoders and decoders based on superstructured fiber Bragg gratings," J. Lightw. Technol., vol. 19, no. 9, pp. 1352-1365, Sep. 2001.

[18] X. Wang, K. Matsushima, K. Kitayama, A. Nishiki, N. Wada, and F. Kubota, "High-performance optical code generation and recognition by use of a 511-chip, 640-Gchip/s phase-shifted superstructured fiber Bragg grating," Opt. Lett., vol. 30, no. 4, pp. 355-357, Feb. 2005.

[19] H. P. Sardesai and A. M. Weiner, "Nonlinear fibre-optic receiver for ultrashort pulse code division multiple access communications," Electron. Lett., vol. 33, no. 7, pp. 610-611, Mar. 1997.

[20] S. Shen, A. M. Weiner, G. D. Sucha, and M. L. Stock, "Bit error rate performance optical CDMA detection under multi-access interference," Electron. Lett., vol. 36, no. 21, pp. 1795-1797, Oct. 2000.

[21] X. Wang and K. Kitayama, "Analysis of beat noise in coherent and incoherent time-spreading OCDMA," J. Lightw. Technol., vol. 22, no. 10, pp. 2226-2235, Oct. 2004.

[22] N. Wada, H. Sotobayashi, and K. Kitayama, "Error-free 100 km transmission of $10 \mathrm{~Gb} / \mathrm{s}$ optical code division multiplexing using BPSK picoseconds-pulse code sequence with novel time-gating detection," IEE Electron. Lett., vol. 35, no. 7, pp. 833-834, Apr. 1999.

[23] H. Sotobayashi, W. Chujo, and K. Kitayama, "1.6-b/s/Hz 6.4-Tb/s QPSK$\mathrm{OCDM} / \mathrm{WDM}(4 \mathrm{OCDM} \times 40 \mathrm{WDM} \times 40 \mathrm{~Gb} / \mathrm{s})$ transmission experiment using optical hard thresholding," IEEE Photon. Technol. Lett., vol. 14, no. 4, pp. 555-557, Apr. 2002.

[24] T. Okuno, M. Onishi, and M. Nishimura, "Ultra-broad-band supercontinuum by dispersion-flattened and decreasing fiber," IEEE Photon. Technol. Lett., vol. 10, no. 1, pp. 72-74, Jan. 1998.

[25] X. Wang, T. Hamanaka, N. Wada, and K. Kiayama, "Dispersion-flattenedfiber based optical thresholder for multiple-access-interference suppression in OCDMA system," Opt. Express, vol. 13, no. 14, pp. 5499-5505, Jul. 2005.

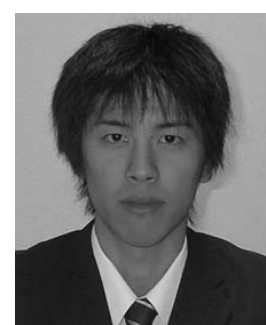

Taro Hamanaka received the B.E. degree in electronics, information systems, and energy engineering from Osaka University, Osaka, Japan, in 2005. He is currently pursuing the M.E. degree at the same university

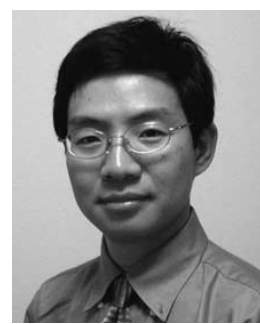

Xu Wang (S'91-M'98) received B.S. degree in physics from the Zhejiang University, Hangzhou, China, the M.S. degree in electronics engineering from the University of Electronics Science and Technology of China (UESTC), Chengdu, China, and the $\mathrm{Ph} . \mathrm{D}$. degree in electronics engineering from the Chinese University of Hong Kong (CUHK), Hong Kong, in 1989, 1992, and 2001, respectively.

From 1992 to 1997, he was a Lecturer in the National Key Laboratory of Fiber Optic Broadband Transmission and Communication Networks of UESTC. From 2001 to 2002, he was a Postdoctoral Research Fellow in the Department of Electronic Engineering of CUHK. From 2002 to 2004, he worked in the Department of Electronic and Information Systems, Osaka University, Osaka, Japan, as TAO Research Fellow. In April 2004, he jointed the Ultra-fast Photonic Network Group of Information and Network Systems Department, National Institute of Communication and Information Technology (NICT), Tokyo, Japan, as an expert researcher. He is also an Adjunct Professor with two universities. His research interests include fiber optic communication networks, optical code division multiplexing, optical packet switching, semiconductor laser, application of fiber gratings, and fiber optic signal processing. He has filed three patents and is the first author of more than 60 technical papers.

Dr. Wang was awarded the Telecommunications Advancement Research Fellowship by the TAO of Japan in 2002 and 2003.

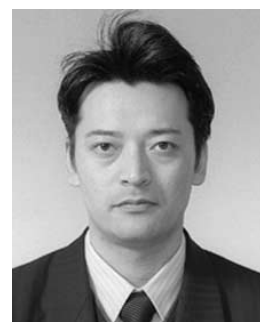

Naoya Wada (M'97) received the B.E., M.E., and Dr.Eng. degrees in electronics from Hokkaido University, Sapporo, Japan, in 1991, 1993, and 1996, respectively.

In 1996, he joined the Communications Research Laboratory (CRL), Ministry of Posts and Telecommunications, Tokyo, Japan. He is currently a Senior Researcher of the National Institute of Information and Communications Technology (NICT), Tokyo, Japan. His current research interests are in the area of photonic networks and optical communication technologies such as optical packet switching (OPS), optical processing, and optical code-division multiplexing (OCDM).

Dr. Wada received the 1999 Young Engineer Award from the Institute of Electronic and Communication Engineers of Japan, and the 2005 Young Researcher Award from Ministry of Education, Culture, Sports, Science and Technology. He is a member of IEEE Comsoc, IEEE LEOS, the Institute of Electronics and Communications (IEICE), the Japan Society of Applied Physics (JSAP), and the Optical Society of Japan (OSJ).

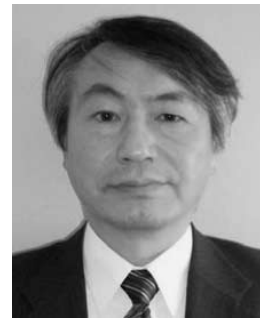

Akihiko Nishiki (M'99) received B.E. and M.E degrees in electrical engineering from Kansai University, Osaka, Japan, in 1981 and 1983, respectively.

He joined Oki Electric Industry Co., Ltd., Tokyo, Japan, in 1983. He has developed fiber Bragg grating devices since 1997 with the Corporate Research and Development Center. His current research interest focusses on the application of fiber Bragg grating to en/decoder for OCDM systems.

Mr. Nishiki is a member of the Japan Society of Applied Physics. 


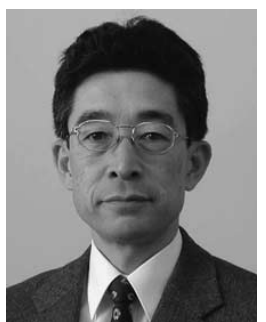

Ken-ichi Kitayama (S'75-M'76-SM'89-F'03) received the B.E., M.E., and Dr.Eng. degrees in communication engineering from Osaka University, Osaka, Japan, in 1974, 1976, and 1981, respectively. In 1976, he joined the NTT Electrical Communication Laboratory. From 1982 to 1983, he spent a year as a Research Fellow at the University of California, Berkeley. In 1995, he joined the Communications Research Laboratory (presently, National Institute of Information and Communications Technology, NiCT), Tokyo, Japan. Since 1999, he has been a Professor with the Department of Electrical, Electronic, and Information Engineering, Graduate School of Engineering, Osaka University. His research interests are in photonic networks and radio-on-fiber communications. He has published over 200 papers in refereed journals, written two book chapters, and translated one book. He holds more than 30 patents.

Dr. Kitayama is a Fellow of IEICE of Japan. He currently serves on the Editorial Boards of the IEEE PHotonics TeChNOLOGy LeTters, IEEE TRANSACTIONS ON COMMUNICATIONS, and Optical Switching and Networking as an Associate Editor. He received the 1980 Young Engineer Award from the Institute of Electronic and Communication Engineers of Japan, the 1985 Paper Award of Optics from the Japan Society of Applied Physics, and the 2004 Achievement Award of IEICE of Japan. 